\title{
Fast micro Hall effect measurements on small pads
}

Østerberg, Frederik Westergaard; Petersen, Dirch Hjorth; Nielsen, Peter F.; Rosseel, Erik; Vandervorst, Wilfried; Hansen, Ole

\section{Published in:}

Journal of Applied Physics

Link to article, DOI:

$10.1063 / 1.3610505$

Publication date:

2011

Document Version

Publisher's PDF, also known as Version of record

Link back to DTU Orbit

Citation (APA):

Østerberg, F. W., Petersen, D. H., Nielsen, P. F., Rosseel, E., Vandervorst, W., \& Hansen, O. (2011). Fast micro Hall effect measurements on small pads. Journal of Applied Physics, 110(3), [033707 ].

https://doi.org/10.1063/1.3610505

\section{General rights}

Copyright and moral rights for the publications made accessible in the public portal are retained by the authors and/or other copyright owners and it is a condition of accessing publications that users recognise and abide by the legal requirements associated with these rights.

- Users may download and print one copy of any publication from the public portal for the purpose of private study or research.

- You may not further distribute the material or use it for any profit-making activity or commercial gain

- You may freely distribute the URL identifying the publication in the public portal 


\title{
Fast micro Hall effect measurements on small pads
}

\author{
Frederik Westergaard Østerberg, ${ }^{1}$ Dirch Hjorth Petersen, ${ }^{1, a)}$ Peter F. Nielsen, ${ }^{2}$ \\ Erik Rosseel, ${ }^{3}$ Wilfried Vandervorst, ${ }^{3,4}$ and Ole Hansen ${ }^{1,5, b)}$ \\ ${ }^{1}$ DTU Nanotech - Department of Micro and Nanotechnology, Technical University of Denmark, \\ building 345 East, DK-2800 Kgs. Lyngby, Denmark \\ ${ }^{2}$ CAPRES A/S, Scion-DTU, Building 373, DK-2800 Kgs. Lyngby, Denmark \\ ${ }^{3}$ IMEC, Kapeldreef 75, B-3001 Leuven, Belgium \\ ${ }^{4}$ Institute voor Kern- en Stralingsfysica, K. U. Leuven, Celestijnenlaan 200D B-3001 Leuven, Belgium \\ ${ }^{5}$ CINF - Centre for Individual Nanoparticle Functionality, Technical University of Denmark, \\ building 345 East, DK-2800 Kgs. Lyngby, Denmark
}

(Received 30 March 2011; accepted 12 June 2011; published online 3 August 2011)

\begin{abstract}
Sheet resistance, carrier mobility, and sheet carrier density are important parameters in semiconductor production, and it is therefore important to be able to rapidly and accurately measure these parameters even on small samples or pads. The interpretation of four-point probe measurements on small pads is non-trivial. In this paper we discuss how conformal mapping can be used to evaluate theoretically expected measurement values on small pads. Theoretical values calculated from analytical mappings of simple geometries are compared to the values found from the numerical conformal mapping of a square onto the infinite half-plane, where well-established solutions are known. Hall effect measurements are performed to show, experimentally, that it is possible to measure Hall mobility in less than one minute on squares as small as $70 \times 70 \mu \mathrm{m}^{2}$ with a deviation of $\pm 6.5 \%$ on a $1 \sigma$ level from accurate reference measurements, when only small sheet resistance variations are present. For samples with a sheet resistance variation of more than $5 \%$, a more time consuming method must be used. Here, the theoretically expected measurement values are computed based on the sheet resistance variation using a finite element method. This more accurate method has an experimental repeatability of $\pm 1.6 \%$ even on inhomogeneous samples.
\end{abstract} (C) 2011 American Institute of Physics. [doi:10.1063/1.3610505]

\section{INTRODUCTION}

In the processing of semiconductor devices, it is becoming increasingly important and difficult to characterize material parameters such as sheet resistance, sheet carrier density, and carrier mobility. These parameters can be characterized through various methods, where some require special sample preparation, and others are destructive. ${ }^{1,2}$ It has previously been shown that micro four-point probes (M4PP) ${ }^{3}$ are able to measure sheet resistance, sheet carrier density, and Hall mobility on thin film samples with at least one insulating boundary. ${ }^{4}$

The existing technique has been experimentally validated for samples with either single or double insulating lateral boundaries, while assuming a uniform sheet resistance throughout the entire sample. This allows for position error suppression $^{5}$ and thus very accurate results are obtained, even when a magnetoresistance contribution is present. ${ }^{6}$ For a more detailed background on micro four-point probe metrology we refer to the recent review by Petersen. ${ }^{7}$

In this paper we investigate the possibility of performing micro Hall effect measurements in less than a minute on small $\left(70 \times 70-100 \times 100 \mu \mathrm{m}^{2}\right)$ test pads, which do not have uniform sheet resistance. The ability to extract the sheet resistance, sheet carrier density, and Hall mobility from

\footnotetext{
${ }^{\text {a)} E l e c t r o n i c ~ m a i l: ~ d i r c h . p e t e r s e n @ n a n o t e c h . d t u . d k . ~}$

${ }^{b)}$ Electronic mail: ole.hansen@nanotech.dtu.dk.
}

small samples is a significant advantage, since these can be patterned in scribe lines on product wafers, and thus the need for expensive test wafers is reduced.

\section{THEORY}

With an applied magnetic flux density, $B_{z}$, normal to the surface of a thin filamentary sample, the current transport in the sample is modified such that the electric field, E, and the sheet current density, $\mathbf{J}_{\mathbf{S}}$, are not parallel. Thus, it follows that the conventional scalar sheet resistance must be replaced by an antisymmetric sheet resistance tensor, $\mathbf{R}_{\mathrm{S}}{ }^{4}{ }^{4}$ with the direct sheet resistance, $R_{0}$, as diagonal elements while the off-diagonal elements are \pm the Hall sheet resistance, $R_{\mathrm{H}}$ $=\mathcal{R}_{\mathrm{H}} B_{z}$, where $\mathcal{R}_{\mathrm{H}}$ is the sheet Hall coefficient. ${ }^{1,4}$

To extract the Hall effect contribution to the four-point probe measurement, it is useful to measure using four different probe configurations $\left(\mathrm{A}, \mathrm{A}^{\prime}, \mathrm{B}\right.$, and $\left.\mathrm{B}^{\prime}\right)$; these are shown in Fig. 1. For configuration $\mathrm{A}$, a current $I_{0}$ is forced through pins 1 and 4 while the potential difference is measured between pins 2 and 3 . In configuration $A$, the measured voltage, $V_{\mathrm{A}}$, can be expressed as, $V_{\mathrm{A}}=\Phi\left(\mathbf{r}_{2}, \mathbf{r}_{1}, \mathbf{r}_{4}\right)-\Phi\left(\mathbf{r}_{3}, \mathbf{r}_{1}\right.$, $\left.\mathbf{r}_{4}\right)$, where $\Phi\left(\mathbf{r}, \mathbf{r}_{+}, \mathbf{r}_{-}\right)$is the electrostatic potential at the point, $\mathbf{r}$, when a current, $I_{0}$, is forced between the inlet, $\mathbf{r}_{+}$, and outlet, $\mathbf{r}_{-}$, positions. The measured voltages for the three remaining configurations can be found in a similar manner. The probe pin spacing is denoted as $s$, as shown in Fig. 1. 


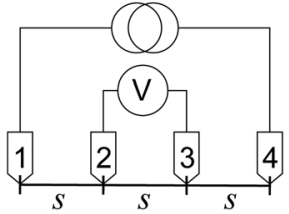

A

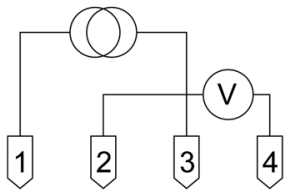

B
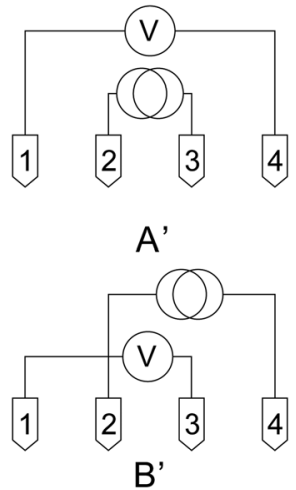

FIG. 1. Illustration of the four different probe configurations $\left(\mathrm{A}, \mathrm{A}^{\prime}, \mathrm{B}\right.$, and $\mathrm{B}^{\prime}$ ) used for M4PP micro Hall effect measurements.

Previous work ${ }^{4}$ has shown that when micro four-point measurements are performed on a thin film with an insulating boundary at $y=0$ and a magnetic flux density, $B_{z}$, applied perpendicular to the thin film, the potential $\Phi\left(\mathbf{r}, \mathbf{r}_{+}, \mathbf{r}_{-}\right)$is given by,

$$
\begin{gathered}
\Phi\left(\mathbf{r}, \mathbf{r}_{+}, \mathbf{r}_{-}\right)=\mathcal{A}_{+} \ln \frac{\left|\mathbf{r}-\mathbf{r}_{-}\right|}{\left|\mathbf{r}-\mathbf{r}_{+}\right|}+\mathcal{A}_{-} \ln \frac{\left|\mathbf{r}-\mathbf{r}_{-}^{*}\right|}{\left|\mathbf{r}-\mathbf{r}_{+}^{*}\right|}+ \\
\frac{I_{0} R_{\mathrm{H}}}{\pi}\left(\arctan \frac{x-x_{+}}{y+y_{+}}-\arctan \frac{x-x_{-}}{y+y_{-}}\right),
\end{gathered}
$$

where

$$
\mathcal{A}_{+} \equiv \frac{I_{0} R_{0}}{2 \pi}\left(1+\frac{R_{\mathrm{H}}^{2}}{R_{0}^{2}}\right), \mathcal{A}_{-} \equiv \frac{I_{0} R_{0}}{2 \pi}\left(1-\frac{R_{\mathrm{H}}^{2}}{R_{0}^{2}}\right),
$$

and $\mathbf{r}_{i}^{*}$ is the mirror image of $\mathbf{r}_{i}$ in the $x$-axis.

Since the potential, $\Phi\left(\mathbf{r}, \mathbf{r}_{+}, \mathbf{r}_{-}\right)$, and therefore the measured voltage, $V_{\mathrm{X}}$, are both proportional to the current, $I_{0}$, a measured transfer resistance can be introduced as $R_{\mathrm{X}}=V_{\mathrm{X}} / I_{0}$, where $\mathrm{X}$ can be $\mathrm{A}, \mathrm{A}^{\prime}, \mathrm{B}$ or $\mathrm{B}^{\prime}$.

It has proved convenient to introduce a mean resistance, $\overline{R_{\mathrm{XX}}}$, and a resistance difference, $\Delta R_{\mathrm{XX}}$, according to the definitions,

$$
\overline{R_{\mathrm{XX}}}=\left(R_{\mathrm{X}}+R_{\mathrm{X}^{\prime}}\right) / 2,
$$

and

$$
\Delta R_{\mathrm{XX}^{\prime}}=R_{\mathrm{X}}-R_{\mathrm{X}^{\prime}}
$$

From the resistance averages, $\overline{R_{\mathrm{AA}^{\prime}}}$ and $\overline{R_{\mathrm{BB}^{\prime}}}$, a pseudo sheet resistance, $R_{\mathrm{P}}$, can be defined as the solution to the modified van der Pauw equation; ${ }^{8,9}$

$$
\exp \frac{2 \pi \overline{R_{\mathrm{AA}^{\prime}}}}{R_{\mathrm{P}}}-\exp \frac{2 \pi \overline{R_{\mathrm{BB}^{\prime}}}}{R_{\mathrm{P}}}=1 .
$$

It has been shown that for $R_{\mathrm{P}}$ in-line position errors are eliminated ${ }^{5,9}$ while $\Delta R_{\mathrm{BB}^{\prime}}$ is insensitive to in-line position errors, ${ }^{5}$ and thus, these two parameters can be measured very accurately. Furthermore when $\left(\mu_{\mathrm{H}} B_{z}\right)^{2} \ll 1, R_{\mathrm{P}}$ and $\Delta R_{\mathrm{BB}^{\prime}}$ are proportional to $R_{0}$ and $R_{\mathrm{H}}$, respectively. These parameters are needed to calculate the Hall mobility using $\mu_{\mathrm{H}}=R_{\mathrm{H}} /\left(R_{0} Z B_{z}\right){ }^{4}$ where $Z= \pm 1$ is the sign of the carrier charge.
Since measurements are not performed on the infinite half-plane where the potential in Eq. 1 is valid, the solution must be transformed to the actual measurement geometry. This may be done by the use of conformal mapping.

\section{A. Conformal Mapping}

Conformal mapping is a useful mathematical tool when a solution to a problem, governed by e.g., a Laplacian differential equation, is known in one geometry (the $z$-plane). ${ }^{10}$ If a conformal mapping, $\left[z=\mathrm{f}(w)\right.$, and $w=\mathrm{f}^{-1}(z)$, where $\mathrm{f}$ is an analytic function] exist between the geometry with a known solution and another geometry (the $w$-plane), the solution in this new geometry can easily be obtained. Here, coordinates in the planes are represented by complex numbers, $z=x+\mathrm{i} y$ and $w=u+\mathrm{i} v$, where the complex unit $\mathrm{i}=\sqrt{-1}$.

In the present case of micro Hall effect measurements, the solution, Eq. (1), is known for the infinite half-plane; thus, if conformal mappings exist between the infinite halfplane and other geometries, the solutions for these can be obtained. The focus of this paper is on measurements performed on square samples, thus only conformal mappings with some relation to squares will be discussed. Thus the square, the infinite strip, and the semi-infinite strip are investigated. These geometries are sketched in Fig. 2, where it is seen that the semi-infinite strip is studied with two different probe orientations. The infinite strip and the semi-infinite strips can be analytically mapped using simple trigonometric functions, whereas analytical mapping of the square to the half-plane involves elliptic functions. ${ }^{11}$ In order to avoid the use of elliptic functions and to demonstrate a method that may be applied to an arbitrary shape, the square is numerically mapped.

\section{B. Analytical Mappings}

The hardest part of analytic conformal mapping is to find the analytic expression that maps one domain onto another. But once the expression is found, it can map the probe positions of a M4PP onto the half-plane and these
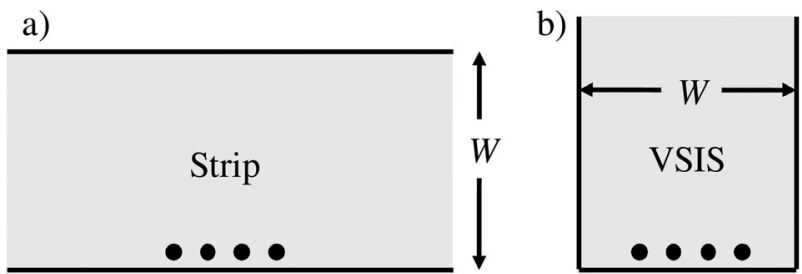

c)

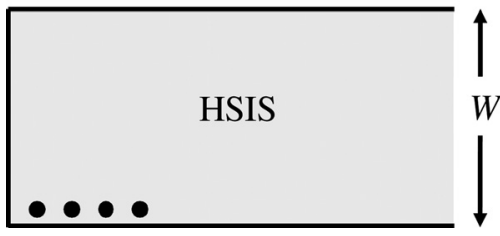

d)

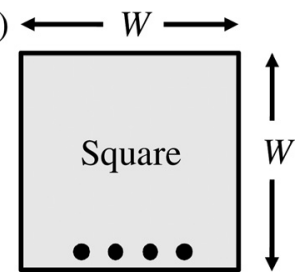

FIG. 2. The four geometries (the infinite strip, the vertical semi-infinite strip VSIS, the horizontal semi-infinite strip HSIS, and the square) studied using conformal mapping. 
positions can be inserted into Eq. (1) to give the potential of the probe configuration. This allows for the computation of the expected pseudo sheet resistance, $R_{\mathrm{P}}$, and resistance difference, $\Delta R_{\mathrm{BB}^{\prime}}$.

\section{Infinite strip}

Next to the infinite half-plane, the infinite strip is the simplest geometry, since just one additional parallel boundary is placed a distance, $W$, from the original half-plane boundary. The conformal mapping pair, $w=(W / \pi) \ln (z)$, $z=\exp (\pi w / W)$, maps the infinite half-plane, $\Im(z)>0$, on the infinite strip, $0<\Im(w)<W$, where $w$ and $z$ are the complex coordinates of the infinite strip and the half-plane, respectively. Thus, the probe positions, $w_{j}$, for $j \in[1,2,3,4]$ on the strip are mapped to the half-plane positions $z_{j}$ by

$$
z_{j}=\exp \frac{\pi w_{j}}{W}
$$

These coordinates may be inserted into Eq. (1) to calculate the resistances, $R_{\mathrm{P}}$ and $\Delta R_{\mathrm{BB}^{\prime}}$.

\section{Semi-infinite strips}

The procedure for theoretically calculating the expected values for measurements on the semi-infinite strips is similar to that on the infinite strip as shown in the previous section, except different mapping functions must be used. The conformal mapping pair, $w=(W / \pi) \arcsin (z)$ and $z=\sin (\pi w / W)$, maps the infinite half-plane, $\Im(z)>0$, on the vertical semiinfinite strip, (VSIS), $|\Re(w)|<W / 2 \cap \Im(w)>0$. The conformal mapping pair, $\mathrm{i} w=(W / \pi) \arcsin (z)$ and $z=\sin (\mathrm{i} \pi w /$ $W$ ), maps the infinite half-plane, $\Im(z)>0$, on the horizontal semi-infinite strip, (HSIS), $|\Im(w)|<W / 2 \cap \Re(w)>0$.

\section{Numerical Mapping}

Numerical conformal mapping may be used for geometries that are difficult or impossible to map analytically. Generalized polygons are geometries, where closed form mapping functions are not readily available, even though the Schwarz-Christoffel conformal mapping scheme defines the derivative of the mapping function. ${ }^{12}$ The polygons, and in particular the square, may then be mapped using a numerical "Schwarz-Christoffel toolbox for MATLAB." 13 In the toolbox the mapping domains are defined and then the toolbox computes a numerical function that works exactly like the analytical mapping functions previously discussed. In this manner, theoretically expected values of $R_{\mathrm{P}}$ and $\Delta R_{\mathrm{BB}^{\prime}}$ for measurements on a square can be calculated. These values may also be calculated using the method of modified images, ${ }^{4}$ where the potential is obtained as a double infinite sum. The values obtained from both approaches have proven to agree.

\section{Comparison of mappings}

The interest in the analytical mapping of the infinite and semi-infinite strips rests with the possibility that these may be used as adequate approximations to replace the numerical mapping of the square. In order to evaluate that possibility,

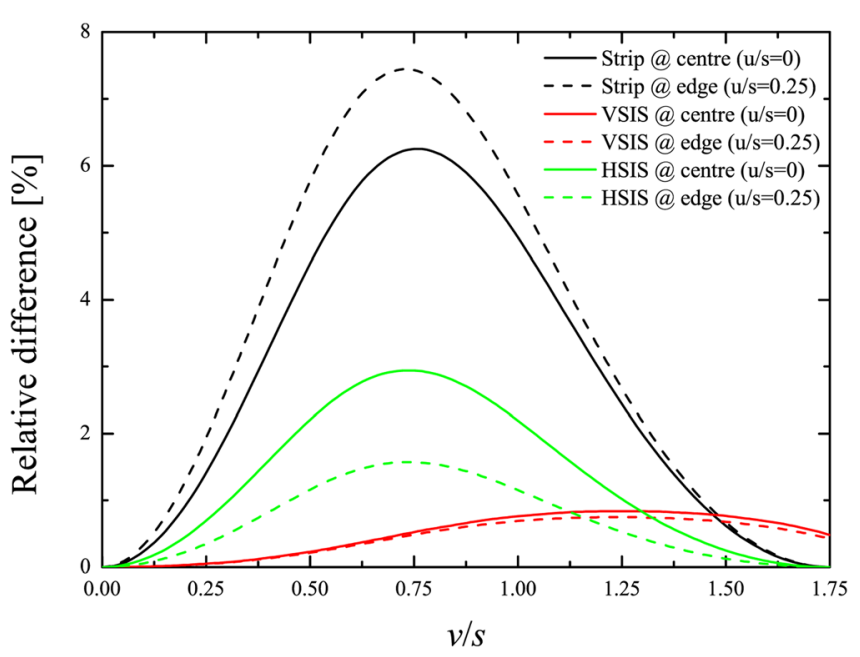

FIG. 3. (Color online) Relative difference (in $\%$ ) between $R_{\mathrm{P}}$ obtained from numerical mapping of a $3.5 \times 3.5 s^{2}$ square and $R_{\mathrm{P}}$ obtained from analytical mappings of infinite and semi-infinite strips as a function of normalized position, $v / s$.

$R_{\mathrm{P}}$ and $\Delta R_{\mathrm{BB}^{\prime}}$, calculated from numerical mapping of a $3.5 \times 3.5 s^{2}$ square are compared to values calculated using the three analytical mappings. The relative differences between the numerical and analytical $R_{\mathrm{P}}$ values are shown in Fig. 3, while the absolute differences between numerical and analytical $\Delta R_{\mathrm{BB}^{\prime}}$ values normalized by $R_{\mathrm{H}}$ are plotted in Fig. 4. From the graphs it is seen that the VSIS resembles the square well close to the boundary at $v / s=0$, while at the center $(v / s=1.75)$ of the square the strip and HSIS are more similar to the square than the VSIS. In general, however, it is necessary to use the full numerical mapping of the correct sample geometry.

\section{EXPERIMENTAL}

The measurements are performed on two shallow trench isolated (STI) patterned wafers; see Fig. 5. Both wafers were

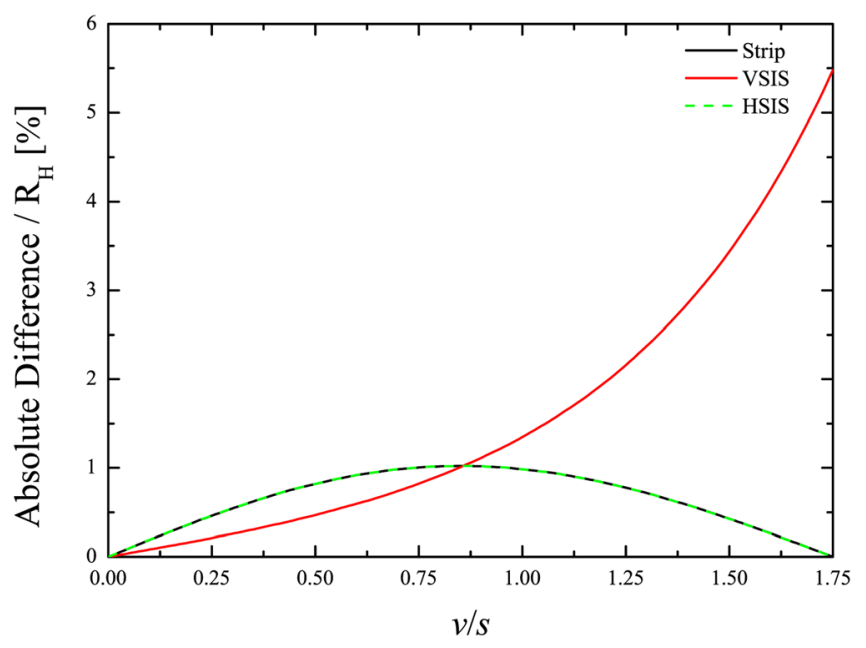

FIG. 4. (Color online) Normalized absolute difference (in \%) between $\Delta R_{\mathrm{BB}^{\prime}}$ obtained from numerical mapping of a $3.5 \times 3.5 s^{2}$ square and $\Delta R_{\mathrm{BB}^{\prime}}$ obtained from analytical mappings of infinite and semi-infinite strips as a function of normalized position, $v / s$. The values shown are normalized by $R_{\mathrm{H}}$. Note that the results for the infinite strip and HSIS cannot be distinguished. 

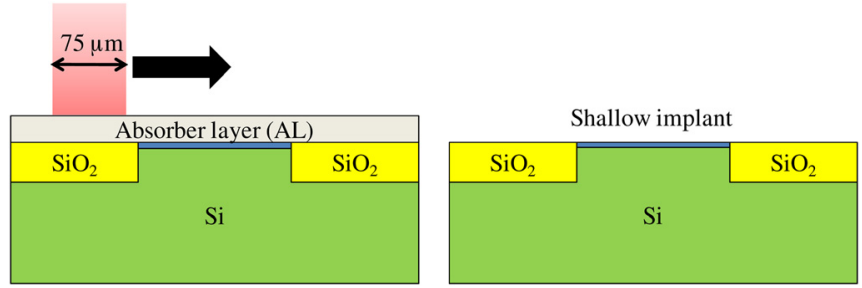

FIG. 5. (Color online) Schematic cross-section of the two shallow trench isolated samples (STI) with and without the absorber layer (400 nm thick). The $\mathrm{SiO}_{2}$ layer is $330 \pm 60 \mathrm{~nm}$ thick. The Applied Materials laser annealer with a $808 \mathrm{~nm}$ diode bar laser has a $75 \mu \mathrm{m}$ wide beam in the scan direction, while it is much wider in the orthogonal direction.

implanted with $10^{15} \mathrm{~cm}^{-2}$ boron ions at $0.5 \mathrm{keV}$. Before annealing, an absorber layer (AL) was deposited on one of the wafers; both wafers were then laser annealed at a nominal temperature of $1200^{\circ} \mathrm{C}$. After the laser anneal the absorber layer was removed in an oxygen plasma.

For the M4PP measurements a CAPRES microRSPM150 system was used. ${ }^{14}$ The M4PPs with an integrated strain gauge were used for accurate surface detection; an optical image of such a M4PP $(s=20 \mu \mathrm{m})$ engaged on a $70 \times 70 \mu \mathrm{m}^{2}$ pad is shown in Fig. 6 .

Two types of M4PP measurements were performed, i.e., sheet resistance measurements and Hall effect measurements. Sheet resistance measurements are used to evaluate sample uniformity, which is important since the existing data treatment for Hall effect measurements rely on a uniform sheet resistance across the sample. In order to accurately measure the sheet resistance it is important to position the probe pins on a mirror axis of the sample to avoid any boundary effects. $^{15}$

The sheet resistance measurements were carried out using a M4PP with a probe spacing of $s=1.5 \mu \mathrm{m}$. The relatively small probe pitch was used in order to enable sheet resistance measurements very close to the boundaries. A sheet resistance scan was obtained by placing the probe on

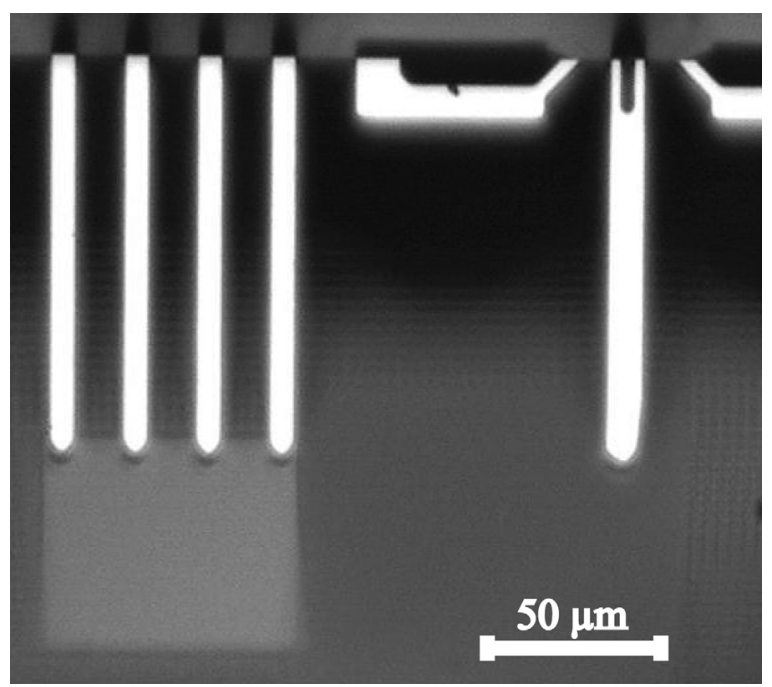

FIG. 6. M4PP engaged on a $70 \times 70 \mu \mathrm{m}^{2}$ pad. The four pins to the left are the M4PP electrodes, while the pin to the right is part of the strain gauge used for surface detection. the mirror axis of the sample, orthogonal to a boundary, followed by a scan parallel to the probe pins through the center of the pad as shown in the left side of Fig. 7.

For the Hall effect measurements it is important to place the probes parallel and close to an insulating boundary and, in addition, apply a magnetic flux density normal to the sample surface, as illustrated in the right side of Fig. 7. For these measurements a probe spacing of $s=20 \mu \mathrm{m}$ was used. The magnetic flux density was applied by using a permanent magnet placed at the center of the sample-chuck, where the sample to be measured was placed immediately above the magnet. The magnetic flux density of the permanent magnet was measured to $0.48 \mathrm{~T}$ using a calibrated Hall sensor.

Measurements were performed on two $70 \times 70 \mu \mathrm{m}^{2}$ pads, and one $100 \times 100 \mu \mathrm{m}^{2}$ pad on each wafer. For the pads on the wafer with AL, all four boundaries were measured, whereas on the wafer without AL, measurements were only performed at the north and south. As a result, 12 Hall mobilities were extracted from the wafer with AL and 6 for the wafer without AL.

Three different fitting routines were used; one where theoretically expected values were fitted to a series of measurements and two routines where only two measurement points were used for fitting. The two-point routines were used to allow for very fast Hall mobility measurements. In the case where a fit to a series of measurements was performed, the position dependent sheet resistance $\left(R_{0}\right)$ was found by least square fitting of a fourth order polynomial to a detailed sheet resistance scan, and then kept fixed during the least square fitting of the Hall sheet resistance $\left(R_{\mathrm{H}}\right)$. Whereas for the least square fitting of the two point methods, both $R_{0}$ and $R_{\mathrm{H}}$ were used as free parameters in the fit. ${ }^{5,16}$

Each of the three fitting routines are described in full detail in Ref. 16, therefore only a brief description of each will be given here. The method where theoretically expected values are fitted to a series of measurements is called the all-inclusive method (AI). It is called all-inclusive because the theoretically expected values are computed based on the experimental sheet resistance scans using the finite element method (FEM) implemented in COMSOL $3.5 ;{ }^{17}$ these values are then fitted to the measured four-point Hall effect scan. The AI method is believed to be the most accurate of the
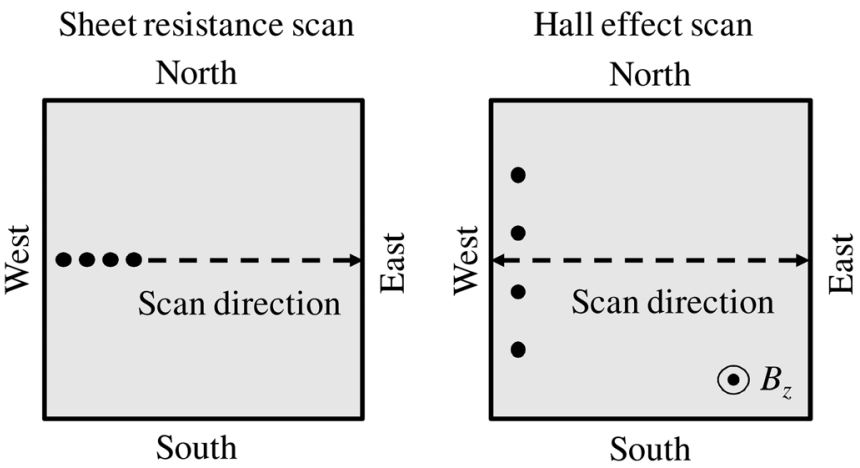

FIG. 7. Probe orientations for sheet resistance scans (left) and Hall effect scans (right). The four boundaries are named North, East, South, and West as shown. 
three methods used, and it is thus used as a reference for the two other methods.

Since the AI method requires both a high resolution sheet resistance scan and a Hall effect scan, the total measurement time is close to $30 \mathrm{~min}$. In many practical applications this method is too time consuming, thus two significantly faster two-point measurement methods are proposed, i.e., the fast-far separation method (FFS) and fastshort separation method (FSS). The names simply refer to the distance between the two measurement points used. For the FFS method, a measurement close to the boundary and a measurement at the center of the pad are used; this method is similar to the fast-3s apart in Ref. 16, however, since the pad used here is small compared to the probes, the fast-3s apart method cannot be used. For the FSS method both measurement points are close to the boundary with a short separation between them; in these experiments, they are separated by 3 $\mu \mathrm{m}$, and the point closest to the parallel boundary is placed 4 $\mu \mathrm{m}$ from the boundary. These numbers have been optimized by simulations using the Monte Carlo method. ${ }^{16}$

\section{RESULTS}

Both sheet resistance and Hall effect scans were done. The sheet resistance scans will be discussed first, since these are used for computing the theoretically expected resistance values used for the AI method.

\section{A. Sheet resistance scans}

Figures 8 and 9 show sheet resistance scans of samples (70 $\mu \mathrm{m}$ and $100 \mu \mathrm{m}$ square pads) on the wafers processed with and without AL, respectively. The letters NS and EW refer to the scan directions used, i.e. NS is north-south and EW is east-west. From the graphs, it is seen that the samples without AL have a significantly larger sheet resistance variation than the samples with AL. The sheet resistance is seen to slightly increase toward the boundaries of the samples with AL, whereas the sheet resistance is signifi-

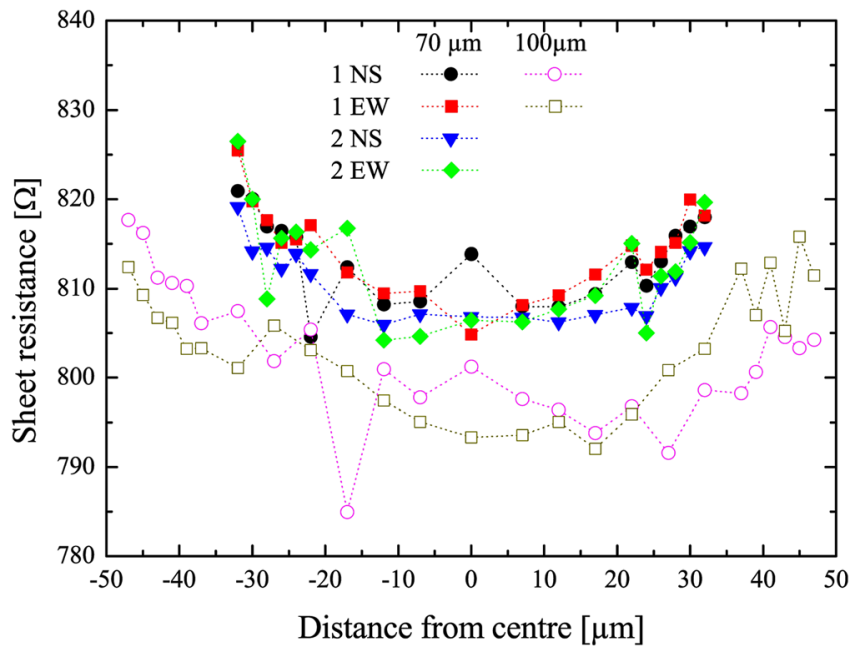

FIG. 8. (Color online) Sheet resistance scans on samples with AL. Scans on two $70 \mu \mathrm{m}$ (filled symbols) and one $100 \mu \mathrm{m}$ (open symbols) square pads in the directions of North-South and East-West are shown.

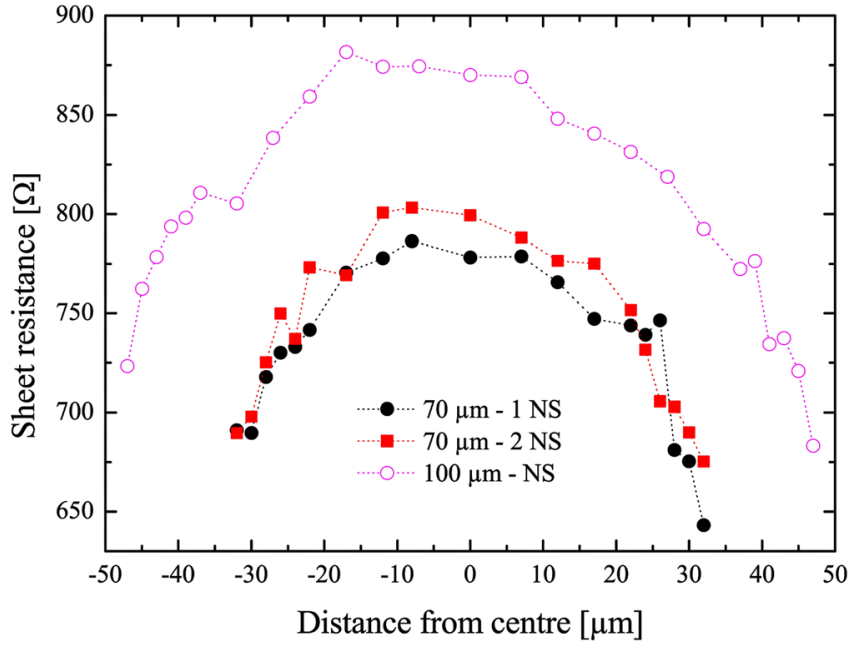

FIG. 9. (Color online) Sheet resistance scans on the samples without AL. Scans on two $70 \mu \mathrm{m}$ (filled symbols) and one $100 \mu \mathrm{m}$ (open symbol) square pads in the direction North-South are shown.

cantly decreasing toward the boundaries on the samples without AL.

\section{B. Hall effect scans-Al method}

The sheet resistance scans shown in Figs. 8 and 9 were used for FEM calculations of the theoretically expected resistance values fitted to the measured resistance values in the AI method. Figures 10 and 11 show examples of measurements and corresponding fits for two $70 \times 70 \mu \mathrm{m}^{2}$ samples without AL. In the graphs the separate sheet resistance scans and a fourth order polynomial fit to the sheet resistance scan are also shown. The fourth order polynomial fit was implemented in the FEM model used for fits to the Hall effect measurements.

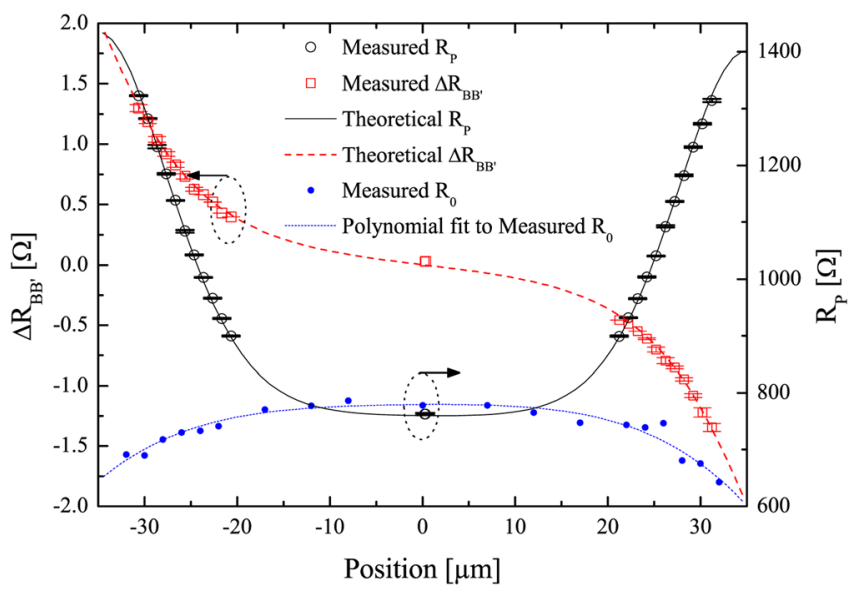

FIG. 10. (Color online) Sheet resistance and Hall effect scans for the first $70 \times 70 \mu \mathrm{m}^{2}$ pad without AL. Results from the separate sheet resistance scan (blue symbol, $\bullet$ ) and a corresponding fourth order polynomial fit (full blue line) are included. Measured $R_{\mathrm{P}}$ (black symbol, $\bigcirc$ ) and $\Delta R_{\mathrm{BB}^{\prime}}$ (red symbol, $\square$ ), are shown along with the resulting AI fits (full black line and dashed red line, respectively). The measurements were performed in the North - South direction. Error bars are also plotted for measured data, but are mostly too small to be distinguished. 


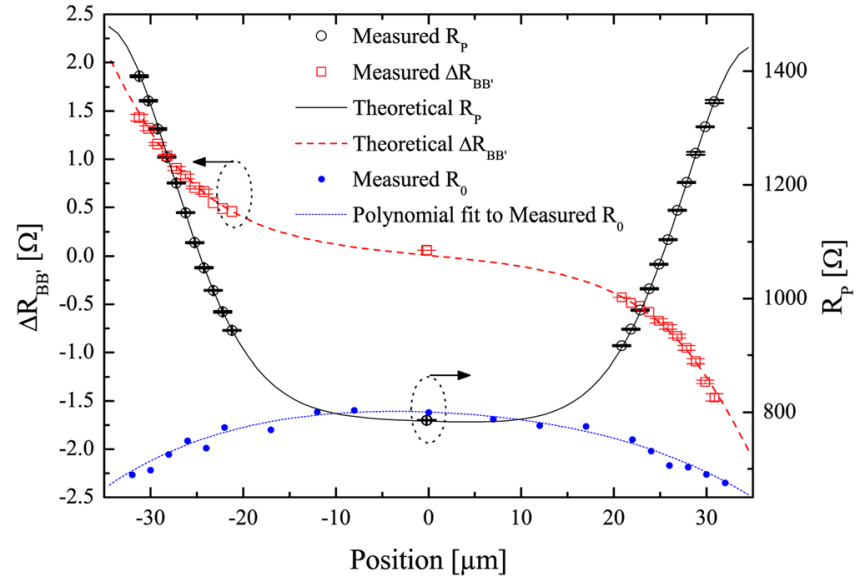

FIG. 11. (Color online) Sheet resistance and Hall effect scans for the second $70 \times 70 \mu \mathrm{m}^{2}$ pad without AL. Results from the separate sheet resistance scan (blue symbol, $\bullet$ ) and a corresponding fourth order polynomial fit (full blue line) are included. Measured $R_{\mathrm{P}}$ (black symbol, $\bigcirc$ ) and $\Delta R_{\mathrm{BB}^{\prime}}$ (red symbol, $\square$ ), are shown along with the resulting AI fits (full black line and dashed red line, respectively). The measurements were performed in the North - South direction. Error bars are also plotted for measured data, but are mostly too small to be distinguished.

\section{Comparison of Hall mobilities}

The extracted mobilities from the three different fitting routines (AI, FFS, and FFS) are shown in Figs. 12 and 13 for the wafer processed without and with $\mathrm{AL}$, respectively. In the graphs the gray bands correspond to $\overline{\mu_{\mathrm{H}}} \pm 3 \sigma_{\mu_{\mathrm{H}}}$ for the AI method. The results are also summarized in Table I.

\section{Detailed scan}

To demonstrate the repeatability and accuracy of the measurement set-up, a more detailed Hall effect scan was done on a $70 \times 70 \mu \mathrm{m}^{2}$ pad with $\mathrm{AL}$. The measurement results are shown in Fig. 14, where fits assuming constant sheet resistance and mobility are also shown.

From the fit, an extracted Hall mobility of $32.2 \mathrm{~cm}^{2} \mathrm{~V}^{-1}$ $\mathrm{s}^{-1}$ results. This Hall mobility value is within one standard deviation of that resulting from the AI method reported in the preceding text.

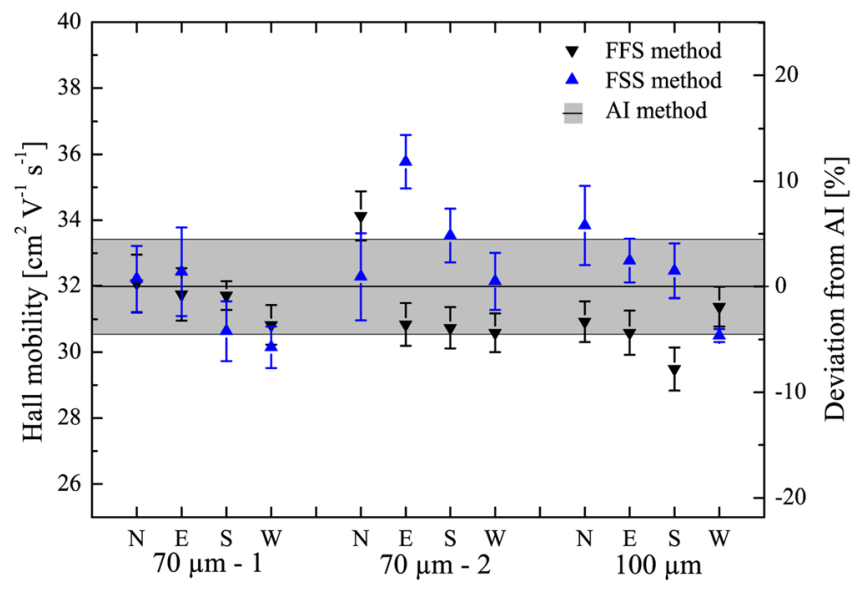

FIG. 12. (Color online) Hall mobilities extracted from the wafer with AL using the three different fitting methods (AI $\boxminus$, FFS $\boldsymbol{\nabla}$, and FSS $\boldsymbol{\Delta}$ ).

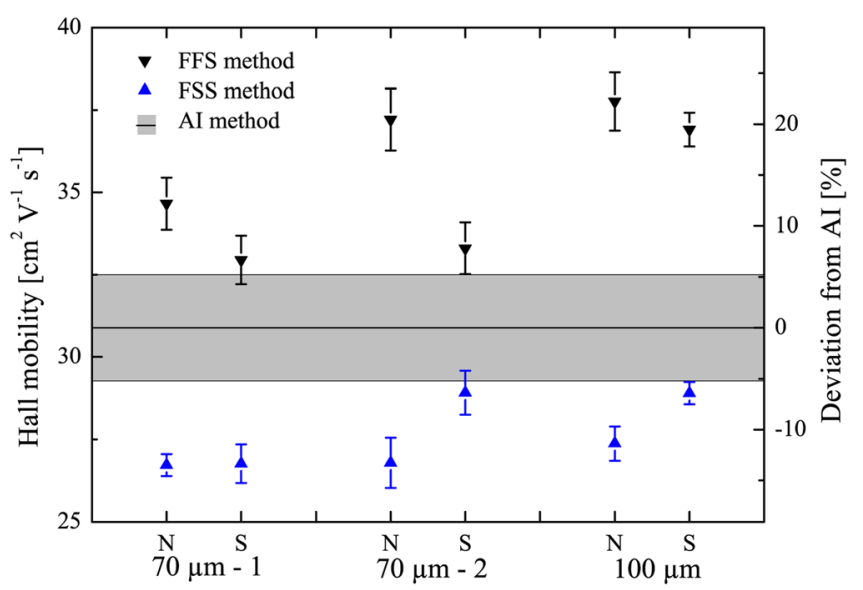

FIG. 13. (Color online) Hall mobilities extracted from the wafer without AL using the three different fitting methods (AI $\boxminus$, FFS $\boldsymbol{\nabla}$, and FSS $\mathbf{\Delta}$ ).

\section{E. Fits using VSIS theory}

For all of the Hall effect results presented in the preceding text, the theoretically expected values have either been computed using FEM or numerical conformal mapping. However, as previously discussed, it would be an advantage to avoid using numerical conformal mapping and use approximate analytical conformal mapping instead. To clarify the feasibility of the approach, the vertical semi-infinite strip was used as an approximation to the square together with the FSS method. We chose VSIS because that is the simplest geometry with the closest resemblance to the square within the experimental range $(0<v / s \leq 1 / 2$, probe within $10 \mu \mathrm{m}$ from the edge) as shown in Figs. 3 and 4. The relative deviation of Hall mobilities extracted using the VSIS approximation from those extracted using numerical conformal mapping of the square can be seen in Table II. In general, the difference is seen to be within $\pm 0.2 \%$, with only three exceptions, where the difference may be as large as $-1.9 \%$.

\section{DISCUSSION}

\section{A. Sheet resistance measurements}

The sheet resistance measurements in Figs. 8 and 9 show significant differences between samples with and without AL. Samples processed without AL have much larger sheet resistance variations toward the sample boundaries than the samples processed with AL. This effect is believed to be caused by optical reflection coefficient differences between the bare silicon surface and the surrounding oxidecovered silicon surface of the samples without AL, which leads to large spatial variations in the effective laser anneal

TABLE I. Extracted Hall mobilities.

\begin{tabular}{lcc}
\hline \hline & $\begin{array}{c}\text { With AL } \\
\overline{\mu_{\mathrm{H}}} \pm \sigma_{\mu_{\mathrm{H}}}\left[\mathrm{cm}^{2} \mathrm{~V}^{-1} \mathrm{~s}^{-1}\right]\end{array}$ & $\begin{array}{c}\text { Without AL } \\
\overline{\mu_{\mathrm{H}}} \pm \sigma_{\mu_{\mathrm{H}}}\left[\mathrm{cm}^{2} \mathrm{~V}^{-1} \mathrm{~s}^{-1}\right]\end{array}$ \\
\hline AI & $31.9 \pm 0.5$ & $30.9 \pm 0.5$ \\
FFS & $31.2 \pm 2.1$ & $35.4 \pm 1.1$ \\
FSS & $32.4 \pm 1.6$ & $27.6 \pm 1.6$ \\
\hline \hline
\end{tabular}




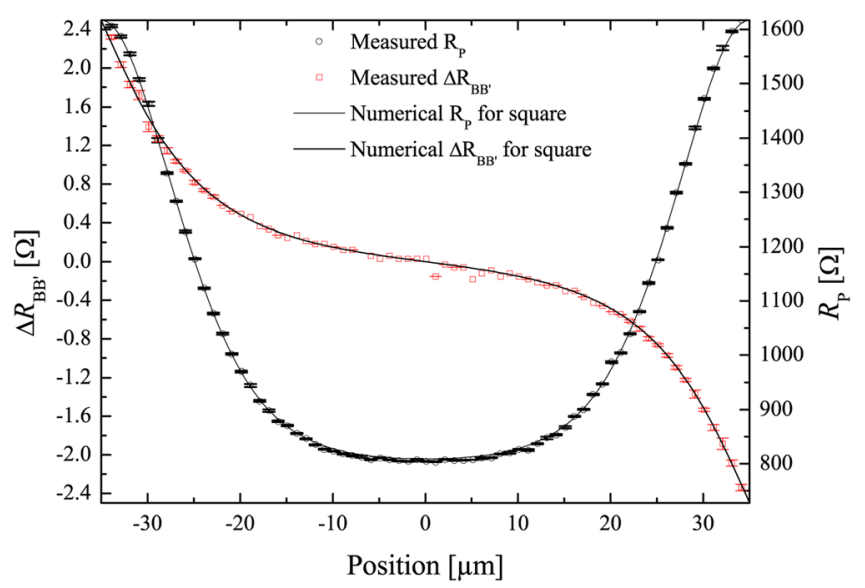

FIG. 14. (Color online) Detailed Hall effect scan on a $70 \times 70 \mu \mathrm{m}^{2}$ pad with AL. Measured $R_{\mathrm{P}}$ (black symbol, $\bigcirc$ ) and $\Delta R_{\mathrm{BB}^{\prime}}$ (red symbol, $\square$ ), are shown along with the resulting fits (full lines). Error bars are also plotted for measured data, but are mostly too small to be distinguished.

temperature across the sample. Samples with AL have a much more uniform optical reflection coefficient and thus a more uniform effective laser anneal temperature. ${ }^{18}$

\section{B. Hall effect measurements}

The Hall effect measurement results show that the fast methods are best suited for the samples with AL, where the sheet resistance variation is smaller than on the samples without AL. The reason for this is that both fast methods rely on uniform sheet resistance and Hall sheet resistance; this condition is not fulfilled on the sample without AL and only approximately met on the sample with AL. For the samples with AL both fast methods produce Hall mobilities that are within $\pm 6.5 \%$ of the mean AI value on a $1 \sigma$ level. The average Hall mobility values extracted using FFS and FSS differ from the average AI value by $-2.2 \%$ and $1.6 \%$, respectively. For the samples without the AL, the FSS method underestimates the Hall mobility by $10.7 \%$ on average, while the FFS method overestimates the Hall mobility by $14.6 \%$ on average, due to the large sheet resistance variation on the samples.

TABLE II. Relative Hall mobility difference resulting from numerical conformal mapping of a square compared to the VSIS approximation. Only the FSS method is used.

\begin{tabular}{lcc}
\hline \hline Boundary & With AL (\%) & Without AL (\%) \\
\hline $70-1 \mathrm{~N}$ & 0.11 & 0.06 \\
$70-1 \mathrm{E}$ & 0.02 & \\
$70-1 \mathrm{~S}$ & 0.17 & 0.08 \\
$70-1 \mathrm{~W}$ & -0.10 & 0.59 \\
$70-2 \mathrm{~N}$ & -1.90 & \\
$70-2 \mathrm{E}$ & -0.05 & 0.09 \\
$70-2 \mathrm{~S}$ & 0.11 & \\
$70-2 \mathrm{~W}$ & 0.07 & 0.70 \\
$100-\mathrm{N}$ & -0.07 & -0.07 \\
$100-\mathrm{E}$ & -0.06 & \\
$100-\mathrm{S}$ & -0.08 & \\
$100-\mathrm{W}$ & -0.15 & \\
\hline \hline
\end{tabular}

The Hall mobilities extracted using the AI method on the two sample types differ by approximately $3.2 \%$, i.e., $31.9 \pm 0.5$ and $30.9 \pm 0.5 \mathrm{~cm}^{2} \mathrm{~V}^{-1} \mathrm{~s}^{-1}$ for the samples with and without AL, respectively. Previous measurements ${ }^{16}$ on larger $430 \times 400 \mu \mathrm{m}^{2}$ pads resulted in Hall mobilities, extracted using the AI method of $31.4 \pm 0.3$ and $32.4 \pm 0.6$ $\mathrm{cm}^{2} \mathrm{~V}^{-1} \mathrm{~s}^{-1}$ for the samples with and without AL, respectively. These differences are both significant considering the measurement accuracy, and understandable considering the effective temperature during the laser anneal; due to the spatial variation in reflectivity of the samples and the width $(75 \mu \mathrm{m})$ of the laser beam, the effective anneal temperature, $T$, differs among the samples such that $T_{400, \mathrm{AL}}>$ $T_{70, \text { noAL }} \sim T_{70, \mathrm{AL}}>T_{400, \text { noAL }}$, as shown in Ref. 18 , the subscripts 400 and 70 refer to sample edge lengths. The active doping concentration increases with increasing the effective anneal temperature, while the mobility decreases with active doping concentration, which is in perfect agreement with the measured Hall mobilities.

The Hall mobilities extracted using analytical conformal mapping of VSIS proved all to be within $2 \%$ of those extracted using the numerical conformal mapping of the square. Most extracted values were even within $0.2 \%$, which is an insignificant error contribution compared to other sources of error. Thus, considerable savings in computational complexity result from replacing numerical conformal mapping by the analytical mapping of VSIS without a significant loss of accuracy. For measurements on samples with an arbitrary shape, however, numerical conformal mapping is extremely useful and versatile.

\section{CONCLUSION}

We have experimentally shown that it is possible to perform fast M4PP Hall effect measurements on small $70 \times 70$ $\mu \mathrm{m}^{2}$ and $100 \times 100 \mu \mathrm{m}^{2}$ square samples and extract the Hall mobility with a deviation of $\pm 6.5 \%$ on a $1 \sigma$ level from the mean AI results, if the samples have sufficiently uniform sheet resistance. Such measurements may be done in less than one minute using the FFS or FSS methods. This is very good considering the small size of the samples and that no additional sample preparation is needed apart from the patterning of the insulating boundaries. For samples with larger sheet resistance variation the two fast methods do not perform equally well, and in such cases the far more time consuming AI methods should be used. The AI method also provides excellent results on uniform samples. With the AI method a repeatability of $\pm 1.6 \%$ can be obtained, which is a factor of 2 improvement over any of the fast methods.

Furthermore, the results suggest that for the FSS method the numerical conformal mapping of a square used in fitting may be replaced by the analytical conformal mapping of a vertical semi-infinite strip with good results and significant savings in computational complexity.

\section{ACKNOWLEDGMENTS}

The authors would like to thank Peter Bøggild and Rong Lin for fruitful discussions and their continuous support. We are grateful for the financial support from the Danish Council 
for Independent Research: Technology and Production Sciences (FTP). The Center for Individual Nanoparticle Functionality (CINF) is sponsored by The Danish National Research Foundation.

${ }^{1}$ D. K. Schroder, Semiconductor Material and Device Characterization (John Wiley \& Sons, New York, 2006).

${ }^{2}$ T. Clarysse, J. Bogdanowicz, J. Goossens, A. Moussa, E. Rosseel, W. Vandervorst, D. H. Petersen, R. Lin, P. F. Nielsen, O. Hansen, G. Merklin, N. S. Bennett, and N. E. B. Cowern, Mater. Sci. Eng., B B154-155, 24 (2008).

${ }^{3}$ C. L. Petersen, T. M. Hansen, P. Bøggild, A. Boisen, O. Hansen, T. Hassenkam, and F. Grey, Sens. Actuators, A A96, 53 (2002).

${ }^{4}$ D. H. Petersen, O. Hansen, R. Lin, and P. F. Nielsen, J. Appl. Phys.104, 013710 (2008).

${ }^{5}$ D. H. Petersen, O. Hansen, R. Lin, P. F. Nielsen, T. Clarysse, J. Goossens, E. Rosseel, and W. Vandervorst, in Proc. 16th IEEE International Conference on Advanced Thermal Processing of Semiconductors, RTP 2008, (IEEE, New York, 2008), pp. 251-256, http://dx.doi.org/10.1109/ RTP.2008.4690563.

${ }^{6}$ D. H. Petersen, O. Hansen, P. Boggild, R. Lin, P. F. Nielsen, D. Lin, C. Adelmann, A. Alian, C. Merckling, J. Penaud, G. Brammertz, J. Goossens, W. Vandervorst, and T. Clarysse, J. Vac. Sci.Technol. B 28, C1C41 (2010).
${ }^{7}$ D. H. Petersen, O. Hansen, T. M. Hansen, P. Boggild, R. Lin, D. Kjaer, P. F. Nielsen, T. Clarysse, W. Vandervorst, E. Rosseel, N. S. Bennett, and N. E. B. Cowern, J. Vac. Sci.Technol. B 28, C1C27 (2010).

${ }^{8}$ L. J. van der Pauw, Philips Tech. 20, 220 (1958).

${ }^{9}$ R. Rymaszewski, J. Phys. E 2, 170 (1969).

${ }^{10}$ R. Schinzinger and P. A. A. Laura, Conformal Mapping: Methods and Applications (Elsevier, New York, 1991).

${ }^{11}$ K. Reck, E. V. Thomsen, and O. Hansen, Opt. Express 19, 1808 (2011).

${ }^{12} \mathrm{~W}$. Kaplan, Introduction to Analytic Functions (Addison-Wesley, Reading, MA, 1966).

${ }^{13}$ T. A. Driscoll, "Schwarz-Christoffel toolbox users guide," Version 2.3, http://www.math.udel.edu/driscoll/software/SC/guide.pdf.

${ }^{14}$ CAPRES A/S, http://www.capres.com.

${ }^{15}$ S. Thorsteinsson, F. Wang, D. H. Petersen, T. M. Hansen, D. Kjær, R. Lin, J.-Y. Kim, P. F. Nielsen, and O. Hansen, Rev. Sci. Instrum. 80, 053902 (2009).

${ }^{16}$ F. Osterberg, D. H. Petersen, F. Wang, E. Rosseel, W. Vandervorst, and O. Hansen, 17th IEEE International Conference on Advanced Thermal Processing of Semiconductors (IEEE, New York, 2009), http://dx.doi.org/ 10.1109/RTP.2009.5373450.

${ }^{17}$ COMSOL, http://www.comsol.com.

${ }^{18}$ E. Rosseel, J. Bogdanowicz, T. Clarysse, W. Vandervorst, C. Ortolland, T. Hoffmann, A. Salnik, L. Nicolaides, S.-H. Han, D. H. Petersen, R. Lin, and O. Hansen, J. Vac. Sci.Technol. B 28, C1C21 (2010). 